Mundo Agrario vol. 18, no 38, e060, agosto 2017. ISSN 1515-5994

Universidad Nacional de La Plata.

Facultad de Humanidades y Ciencias de la Educación.

Centro de Historia Argentina y Americana

\title{
Procesos organizativos y políticas públicas destinadas a productores familiares del sur del Area Metropolitana (provincia de Buenos Aires, Argentina), 2002-2015
}

Organizational processes and public policies for family producers in the south of the Metropolitan Area (Buenos Aires-Argentina), 20022015

\section{Cecilia Seibane *, Guillermina Ferraris *}

\section{PALABRAS CLAVE}

Políticas públicas

Agricultura familiar

Buenos Aires

Organizaciones

\section{KEYWORDS}

Political

Farming family

Buenos Aires

Organizations
* Departamento de Desarrollo Rural, Facultad de Ciencias Agrarias y Forestales, Universidad Nacional de La Plata, Argentina | ceciseibane@hotmail.com; guillerminaferraris@hotmail.com

\section{RESUMEN}

El objetivo del trabajo es caracterizar a las organizaciones de pequeños productores familiares en el sur del Área Metropolitana de Buenos Aires (AMBA Sur), en los partidos de La Plata, Berazategui y Florencio Varela. La metodología utilizada es de tipo cualitativo. Los resultados muestran que las políticas públicas implementadas hacia el sector hasta el año 2015 promovieron instancias que facilitaron la organización y el fortalecimiento de estos productores, dando lugar a distintas formas asociativas. La tierra y la comercialización son problemáticas muy sentidas. En la actualidad, estas organizaciones participan en mesas regionales, generando y fortaleciendo instancias organizativas territoriales cada vez más articuladas. 


\section{Introducción}

El Cinturón verde del Gran La Plata se encuentra en el Área Metropolitana Sur de Buenos Aires (AMBA Sur), e incluye a un conjunto de partidos ubicados en la provincia de Buenos Aires, hacia el Sur de la Ciudad Autónoma de Buenos Aires. Entre ellos, relacionados con la actividad hortiflorícola, se destacan La Plata, Berazategui, Florencio Varela, los cuales constituyen uno de los cinturones verdes más importantes del país, y que además abastece con alimentos frescos y flores a toda el área metropolitana de Buenos Aires.

Figura 1

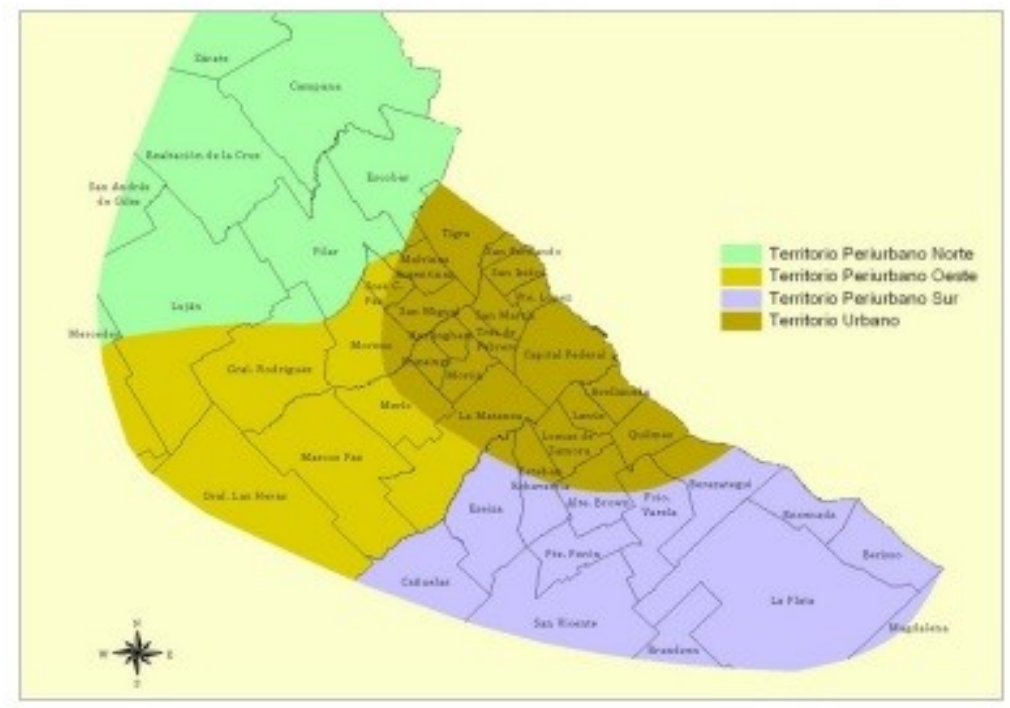

Fuente INTA (2008)

El desarrollo de los sistemas de producción en el área hortiflorícola se ha producido a partir de un conjunto de tecnologías que han permitido incrementar los niveles de productividad a partir de la intensificación en la utilización de los recursos naturales y la mano de obra. En relación con este último punto, la producción en la zona ocupa en término medio 1,5 personas /ha/año en el caso de sistema al aire libre o a campo, y 4 personas/ha/año en el sistema bajo cubierta (García y Kebat, 2008; INTA, 2005).

El avance de los procesos de periurbanización alrededor de las grandes ciudades pone en tensión a la producción, ya que el valor de la tierra se ha incrementado tanto para la compra como para el arrendamiento (Ringuelet, 2008; García, 2008; Merchán, 2016).

Por otro lado, la producción bajo invernáculos que se desarrolla desde comienzos de la década de los '90 se realizó a partir de la adopción de un conjunto de tecnologías biológicas y químicas, muchas de ellas importadas, cuyo su valor se cotiza en dólares (Vega, 1999).

Luego de la crisis del año 2001, se generaron en Argentina profundos cambios y transformaciones que llevaron además a la crisis de representatividad de entidades de productores que existían por aquel entonces en la zona de estudio. Si bien existen en la zona organizaciones de productores con una vasta trayectoria, se visualizan en los últimos 10 años procesos organizativos que se desarrollan con gran intensidad e involucran a un conjunto heterogéneo de actores en un contexto de políticas de Estado que promovieron la organización de los mismos. En esos procesos los pequeños agricultores familiares desarrollaron distintos modos de participación para el abordaje de complejas situaciones que se les presentan y que tienen como denominador común el acceso a la tierra y la comercialización. Esta situación implicó nuevas formas de acercamiento y gestión por parte de las instituciones para la ejecución de políticas destinadas al sector.

La pregunta de investigación que nos guía es: ¿qué características tienen los procesos organizativos que se visualizan en la región estudiada? 
La hipótesis de trabajo es que las políticas de desarrollo implementadas por el Estado en la zona estudiada a partir del año 2002 podrían haber contribuido a la promoción y el fortalecimiento de las distintas formas organizativas.

El objetivo del trabajo es caracterizar las organizaciones de pequeños productores familiares que vienen protagonizando procesos de participación y fortalecimiento en la última década, considerando su año de origen, cantidad de productores, y principales demandas, así como también el modo en que han influido las motivaciones, intereses y cambios contextuales.

La metodología utilizada es de tipo cualitativa, lo que permitió una mejor comprensión de la temática abordada. Se trabajó con recopilación bibliográfica, análisis de documentos institucionales y entrevistas a productores y técnicos relacionados con la temática.

\section{Estado, políticas públicas y organizaciones hortícolas}

Los datos del Censo hortiflorícola de Buenos Aires 2005 (CHFBA), en relación con la tenencia de la tierra para la zona bajo estudio, muestran que más del $60 \%$ de los productores son arrendatarios y llevan a cabo la administración de los establecimientos productivos; además, trabajan junto a su familia, aun cuando están en condiciones de contratar mano de obra .

Esta nueva configuración social y productiva puso en la agenda de las instituciones del sector público un cambio de enfoque en el abordaje de la realidad, hacia una perspectiva más integral, con un enfoque territorial en el que, entre otros objetivos, se puede señalar el fortalecimiento organizacional. Por ello, hubo un cambio en las políticas dirigidas a este conjunto de actores sociales enmarcados en la Agricultura Familiar (AF).

\subsection{Acerca de organizaciones sociales y participación}

Existen numerosos aportes desde los estudios académicos sobre organizaciones sociales, de entre ellos, coincidimos con Fournier, Hantouch, Spampinato, Testa y García (2012): "Son una referencia de participación social, son actores que inciden en la realidad y a la vez, escenario de encuentros y desencuentros donde la propia realidad se manifiesta en toda su dimensión” (p. 15).

Asimismo, señalan la importancia de estudiar el contexto sociohistórico en el que mutuamente se determinan, analizando el rol que cumplen, qué tipo de interacciones tienen entre ellas y con otras instancias de la sociedad, cómo se paran frente al poder.

Es importante realizar algunas consideraciones acerca de la identidad, tema vinculado a los procesos organizativos. Para Melucci (1999) la identidad es un proceso dinámico que se construye permanentemente, es el resultado de una acción colectiva. Según este autor, la identidad colectiva es interactiva y compartida, producida por varios individuos y que concierne a las orientaciones de acción y el ámbito de oportunidades y restricciones en el que tiene lugar la acción. Por interactiva y compartida entiende que debe concebirse como un proceso, porque se construye y negocia a través de la activación repetida de las relaciones que unen a los individuos. Los productores organizados se identifican como agricultores familiares, debido a que tienen como principal objetivo la reproducción social de la familia en condiciones dignas. Los movilizan el acceso a la tierra, condiciones justas de comercialización, y es en torno a estos tópicos que se organizan.

Por otro lado seguiremos a Cardareli y Rosenfeld (1998) que describen el proceso de participación como:

...la conjunción de dos movimientos básicos de ida y vuelta: la activación de la población o powerment para negociar con actores locales y de otros niveles, a partir de intereses específicos, generando y fortaleciendo instancias organizativas territoriales cada vez más articuladas y la formación y sustentabilidad de modalidades de gestión asociada que se instalen con permanencia y lleven a la práctica el principio de la representación, con ampliación progresiva del conjunto de actores e instituciones comprometidas... (p. 136). 
En el mismo sentido, diversos autores hacen referencia a diferentes niveles de participación: información, opinión y toma de decisiones. Diferencian la participación real, basada en la influencia social y en la toma de decisiones, de la participación simbólica, en la que se ejerce un grado mínimo de influencia. (Robirosa, Cardarelli y Lapalma , 1995).

A partir de distintas problemáticas sentidas por la agricultura familiar en esta región, se han dado diversas formas de participación que contribuyeron a la organización del sector.

\subsection{Políticas de desarrollo}

En relación con las políticas de desarrollo llevadas a cabo por instituciones del sector público en la zona bajo estudio existen un conjunto de antecedentes documentados en distintas fuentes, disponibles en versión impresa y en la web. En este trabajo el interés se focalizó en aquellas implementados luego del año 2002, y que tuvieron como principal destinatario a los pequeños agricultores familiares.

En el año 2003, el INTA comenzó el Programa Federal de Apoyo al Desarrollo Rural Sustentable (ProFeder), e integró al programa Cambio Rural como un instrumento más de intervención en el marco de una estrategia consensuada con los actores del territorio (Programa Cambio Rural, 2010). De este modo, se amplió el accionar de Cambio Rural (que existía desde 1993) manteniendo la metodología de trabajo grupal pero dando respuesta a las nuevas demandas de las pymes en materia de innovación tecnológica y organizacional, de gestión y de información, adecuadas a cada realidad local/ territorial. En la región AMBA sur se da respuesta a la demanda de asesoramiento técnico por parte de los productores hortícolas a través de este programa (Feito, 2005; Ringuelet y Garat, 2000). Con el relanzamiento del programa en el año 2014 como Cambio Rural II, se profundiza la intervención en la región y se prioriza la demanda de las organizaciones. A partir de entrevistas realizadas a agentes del INTA en la zona se pudo corroborar la aprobación de 40 grupos de productores hortíflorícolas.

En el marco del Programa Nacional de Investigación y Desarrollo tecnológico para la Pequeña Agricultura Familiar del INTA se creó en 2005 el Centro de Investigación y Desarrollo Tecnológico para la Pequeña Agricultura Familiar (CIPAF) integrado por cinco institutos de acción regional (IPAF). Su objetivo es generar, adaptar y validar tecnologías apropiadas para el desarrollo sostenible de la pequeña agricultura familiar. El IPAF Región Pampeana tiene su ámbito de acción en distintas provincias; su localización en el partido de La Plata posibilita el desarrollo de acciones dirigidas al cinturón hortiflorícola. (Dumrauf et al, 2012)

Para el año 2006, el Instituto Nacional de Tecnología Agropecuaria (INTA) implementó el Programa Nacional de Apoyo al Desarrollo de los Territorios (PNTER), que muestra un grado de apertura inédito de la institución hacia las temáticas territoriales. Ese mismo año financió un proyecto integrado de grandes dimensiones que se centra fundamentalmente en estudiar aspectos tecnológicos y de impacto ambiental de la horticultura en el espacio de influencia metropolitano de Buenos Aires. (Barsky, 2011)

Con la participación de Argentina en la Red de Agricultura Familiar (REAF), se comenzó con el proceso de identificación y reconocimiento de las organizaciones de agricultores familiares existentes en el país. Éste fue el paso previo a la creación de una entidad de segundo grado que, en el 2006, se convertiría en el Foro Nacional de Organizaciones de la Agricultura Familiar (FORO); este espacio fue la articulación de lo público, el Estado Nacional, con lo privado, las organizaciones de la AF. Con la creación del FORO, el espacio de participación de los AF se amplió significativamente; se pasó de una representación de grupos en las Unidades Provinciales, a una entidad formalmente constituida y reconocida, que estaba en condiciones de peticionar al Estado (Bertoni y Soverna, 2014).

También a instancias del FORO se creó en 2008 el Registro Nacional de la Agricultura Familiar (RENAF). El Registro tiene como objetivo confeccionar un padrón de Agricultores Familiares, contar en forma oportuna y permanente con información sobre los mismos, y ofrecer una identificación genuina a los AF que les facilitaría acceder a las políticas que se implementaren (Bertoni y Soverna, 2014). 
En el año 2007, la Provincia de Buenos Aires incluye por primera vez en su organigrama una Dirección de Agricultura Periurbana, bajo la órbita de la Subsecretaría de Asuntos Agrarios. (Barsky, 2011)

Durante el año 2008, el Servicio Nacional de Sanidad y Calidad Agroalimentaria (SENASA) realizó la apertura del Registro Nacional de Productores Agropecuarios (RENSPA) y comenzó sus primeras acciones en horticultura periurbana (Barsky, 2011). En el año 2009 se creó -en la órbita del SENASA- la SENAF, Comisión de Agricultura Familiar del SENASA, espacio constituido por organismos públicos, universidades y organizaciones de productores, que busca construir de manera participativa nuevas normas que regulen la producción de alimentos y adecuar las normas vigentes contemplando las características propias del sector.(SENASA; 2016)

Otra política iniciada en esta etapa y de relevancia para la AF fue la creación del Monotributo Social Agropecuario a costo cero, a través de un convenio con el Ministerio de Desarrollo Social de la Nación que comparte los costos con el Ministerio de Agricultura. (Bertoni y Soverna, 2014)

La Subsecretaria de Desarrollo Rural y Agricultura Familiar (SsDRyAF), dependiente de la entonces Secretaria de Agricultura y Pesca de Nación, instrumentó en el año 2008 el plan de apoyo a pequeños y medianos productores (PAPyMP), que permitió durante varios años la transferencia de fondos a familias, comunidades, organizaciones y a entidades públicas. (Bertoni y Soverna,2014).

En cuanto a la vinculación con las organizaciones de la AF, se produjeron dos cambios importantes a destacar. Se incorporaron como beneficiarias directas de las políticas y se institucionalizó el espacio de participación. Durante la instrumentación del PAPyMP se atendió a una gran diversidad de demandas planteadas por organizaciones, municipios y provincias. El financiamiento directo a organizaciones y la consideración de provincias y municipios como actores del desarrollo rural es otra novedad de la etapa. (Bertoni y Soverna, 2014)

El Programa de Fortalecimiento de los periurbanos fue un lineamiento de política que surgió en 2008 impulsado por el Ministerio de Agricultura de Nación, que se plasmó en el convenio suscripto junto con los municipios, y que tenía como objetivos modernizar la cadena de abastecimiento hortiflorícola para reducir el impacto sobre el medio ambiente, mejorar la calidad higiénico-sanitaria de los productos, generar mayor valor agregado y una adecuada distribución del mismo (Barsky, 2011).

Así, en La Plata, con la participación de un equipo compuesto por la Municipalidad de La Plata, el Ministerio de Asuntos Agrarios de la Provincia de Buenos Aires, el INTA, el Ministerio de Agricultura de Nación y la Facultad de Ciencias Agrarias y Forestales se definieron los principales lineamientos y estrategias de intervención para lograr los objetivos deseados (Seibane et al, 2014).

En Florencio Varela, en el año 2002, se creó el Instituto de Desarrollo Económico local, un organismo municipal que buscó promover el desarrollo del sector productivo implementando programas relacionados con la actividad hortiflorícola, entre ellos, el programa periurbano.

En relación con el Ministerio de Asuntos Agrarios de la Provincia de Buenos Aires, a partir de entrevista realizada a un técnico de la Dirección de Horticultura, Floricultura y Fruticultura, se pudo comprobar el trabajo realizado en cuanto a la implementación de políticas relacionadas con el sector, que articulaba con los Municipios y el INTA local, y focalizaba las acciones en la zona a partir de la temática de buenas prácticas agrícolas para la horticultura.

Con respecto a la provincia de Buenos Aires, y en relación con la zona bajo estudio, se debe mencionar a Cambio Rural Bonaerense (CRB), un programa de promoción del asociativismo similar al de Cambio Rural impulsado por el INTA una década antes. Desde 2002, CRB realizó trabajos de asistencia técnica a la horticultura en distintos partidos, entre ellos, La Plata, Berazategui y Florencio Varela, hasta el año 2006 (Feito, 2007).

Durante el año 2011 se aprobaron los lineamientos estratégicos para la Subsecretaria de Agricultura Familiar (SsAF), entre los que se incluyeron la democratización del acceso a los alimentos con fuerte vinculación a la seguridad alimentaria, el fortalecimiento de las organizaciones de la agricultura familiar, tierra, hábitat, territorios, 
pueblos originarios y agregado de valor en origen. Sostenidos por esos lineamientos estratégicos se incorporaron proyectos de fortalecimiento de las organizaciones, se trabajó en la promoción de la FONAF como Federación de Organizaciones de la Agricultura Familiar, que pasó a ser una organización estrictamente gremial, a diferencia del Foro, en el que había participación público-privada. En materia de tierras, se trató de "dar respuesta a los agricultores familiares, campesinos e indígenas, sobre los legítimos reclamos de acceso a la tierra” (Bertoni y Soverna, 2014).

La conducción de la Subsecretaria de Agricultura Familiar (SsAF), que asumió en agosto de 2012, terminó de definir los lineamientos estratégicos a los que denominó soberanía alimentaria (que incluye producción, comercialización y agregado de valor), cualificación de la producción artesanal de los AF, y tierra, agua y hábitat y dignificación del campesino y el trabajador de los sistemas productivos.

Luego, de acuerdo al Boletín Oficial de la República Argentina, en el Decreto 1030 (2014) se presentaba la creación de la Secretaria de Agricultura Familiar (SAF) con un amplio conjunto de objetivos.

El Foro de Universidades para la Agricultura Familiar de la Región Pampeana, creado en 2009 por el IPAF, es un ámbito en el que participan distintas Universidades , que comenzaron a pensar y generar acciones para el sector en diversas líneas tales como el acceso al agua, energías renovables, transición agroecológica, comercialización y acceso a tierras .(IPAF, Documento 1)

\subsection{Organizaciones de productores en la región estudiada}

El sector hortiflorícola como sector productivo y económicamente significativo para la región se encuentra organizado desde mediados del siglo XX en pos de bregar por mejores condiciones de producción y comercialización.

De acuerdo a INTA (2005), en el diagnóstico participativo realizado con actores de la cadena hortícola del área de influencia del Centro Regional Buenos Aires Norte, una de las debilidades que se presentaba era el bajo nivel de organización de los productores en ese entonces.

Lo que se aprecia en la actualidad es un conjunto de organizaciones de productores que se han formado en su gran mayoría en los últimos 10 años y, en menor proporción, otras que se fueron conformando con anterioridad y que hoy continúan activas.

A partir de la recopilación bibliográfica y del trabajo realizado en terreno se han podido identificar 29 organizaciones activas. De acuerdo con ello, se presenta la información en dos tablas. La Tabla 1 muestra un conjunto de organizaciones de productores que se originaron antes del año 2000 en distintos contextos sociohistóricos que los han llevado a organizarse y que aún continúan vigentes. Han desarrollado un conjunto de acciones con distintos objetivos, entre ellos, defender los intereses de los productores que representan, proveer insumos y brindar un espacio para la comercialización de la producción. Estos datos fueron relevados en entrevistas a informantes claves.

La Asociación de Productores Hortícolas de La Plata y Aphovabe, que nuclean a los viejos quinteros de la zona que en su mayoría son descendientes de inmigrantes europeos- son ambas entidades de carácter gremial.

En 1987, surge formalmente la Asociación de Medieros y Afines (ASOMA), su finalidad era conseguir tierras y herramientas para trabajar, y mejorar así la crítica situación social de los medieros y de otros sectores sociales (Valtriani y Velarde, 2001). 
Tabla 1: Organizaciones activas de productores La Plata, Berazategui y Florencio Varela con origen hasta el año 2000

\begin{tabular}{|c|c|c|}
\hline ORGANIZACIÓN & $\begin{array}{l}\text { Año de Origen- } \\
\text { Localidades }\end{array}$ & Cantidad productores//familias \\
\hline $\begin{array}{l}\text { Asociación de productores hortícolas } \\
\text { de La Plata }\end{array}$ & $\begin{array}{l}\text { 1984- } \\
\text { La Plata }\end{array}$ & $\begin{array}{l}150 \text { productores. Organización de } \\
\text { productores tradicionales, descendientes } \\
\text { de inmigrantes }\end{array}$ \\
\hline $\begin{array}{l}\text { Asociación de Productores } \\
\text { Hortiflorícolas de Florencio Varela y } \\
\text { Berazategui (Aphovabe) }\end{array}$ & $\begin{array}{l}\text { 1995- } \\
\text { Florencio Varela } \\
\text { Berazategui }\end{array}$ & 180 productores \\
\hline $\begin{array}{l}\text { ASOMA, Asociación de Medieros y } \\
\text { Afines }\end{array}$ & $\begin{array}{l}1987- \\
\text { La Plata y partidos } \\
\text { vecinos }\end{array}$ & 580 productores, medieros y peones \\
\hline Cooperativa Argentina de Floricultores & $\begin{array}{l}1975- \\
\text { La Plata y partidos } \\
\text { vecinos }\end{array}$ & 600 productores florícolas \\
\hline $\begin{array}{l}\text { APF, Cooperativa Asociación } \\
\text { Productores Familiares de Florencio } \\
\text { Varela }\end{array}$ & $\begin{array}{l}\text { 1995- } \\
\text { Fcio. Varela }\end{array}$ & 20 productores \\
\hline MERCOFLOR & $\begin{array}{l}\text { 1998- } \\
\text { La Plata y partidos } \\
\text { vecinos }\end{array}$ & 200 productores florícolas \\
\hline
\end{tabular}

El origen de la Cooperativa Argentina de Floricultores y Mercoflor se da por causa de la necesidad de instalar un mercado para la comercialización de flores. Además, en el caso de la cooperativa, la misma provee insumos a los productores. Cabe aclarar que la región concentra la mayor cantidad de productores florícolas del país. La Cooperativa Asociación Productores Familiares de Florencio Varela (APF) es producto del trabajo realizado por $\mathrm{CEDEPO}^{1}$ desde la década del '90, con varias familias de la zona a las que ya se les venía dando asesoramiento individual. En algunos casos, es a partir del trabajo conjunto de CEDEPO con los agricultores - sobre todo con las mujeres-, que éstos se reconocen como productores de alimentos y eligen profundizar esa vía como forma de sostenimiento (Hindi, 2015).

El INTA, en el marco de un enfoque de desarrollo territorial, reformuló su estrategia de intervención y comenzó a reorganizarse. En 2007 y 2008, la temática del periurbano adquiere relevancia en la agenda institucional, y se genera una importante discusión interna en la que la inquietud principal era si el INTA debería involucrarse en la gestión del sector agropecuario del AMBA. En 2009, el Centro Regional Buenos Aires Norte (del cual depende el Área Metropolitana) trabajó en el armado de la estructura, y en 2010 se realizó el lanzamiento del INTA EEA AMBA. 
Tabla 2: Organizaciones actuales de productores de La Plata, Berazategui y Florencio Varela con origen luego del año 2001

\begin{tabular}{|c|c|c|}
\hline ORGANIZACION & Año Origen-Localidades & $\begin{array}{l}\text { Cantidad } \\
\text { productores//familias }\end{array}$ \\
\hline APF el Guadalquivir & 2010 - El Peligro y El Pato & 130 productores \\
\hline $\begin{array}{l}\text { Asociación Civil: } \\
\text { Colectividad Boliviana del } \\
\text { Sur }\end{array}$ & 2000 - Fcio. Varela & 30 productores \\
\hline Asociación 15 de abril & 2015 - Abasto Romero & 60 productores \\
\hline Asociación Tierra Fértil & 2015 - Arturo Seguí y Abasto & $\begin{array}{l}30 \text { productores hortícolas y } \\
\text { florícolas }\end{array}$ \\
\hline Asociación 1610 & 2009 - Fcio. Varela, La Capilla & 20 productores hortícolas \\
\hline $\begin{array}{l}\text { Asociación de Productores de } \\
\text { la Economía Regional }\end{array}$ & 2013 - Parque Pereyra & 52 productores \\
\hline Asociación La Primavera & 2011 - El Peligro y El Pato & 90 productores \\
\hline $\begin{array}{l}\text { Asociación Pioneros de mi } \\
\text { Tierra }\end{array}$ & 2013 - Ángel Etcheverry, Olmos y Abasto & 80 productores hortícolas \\
\hline $\begin{array}{l}\text { Asociación Platense de } \\
\text { Horticultores Independientes. }\end{array}$ & 2005 - Olmos, Abasto y Ángel Etcheverry & Más de 100 productores \\
\hline Asociación San Roque & 2015 - Olmos, Abasto y Ángel Etcheverry & 40 productores \\
\hline $\begin{array}{l}\text { Asociación de productores } \\
\text { Las Banderitas }\end{array}$ & $\begin{array}{l}2010 \text { - Banderitas, Colonia Urquiza y } \\
\text { Abasto }\end{array}$ & 21 productores de flores \\
\hline $\begin{array}{l}\text { Cooperativa } \\
\text { de Trabajo la Unión Limitada }\end{array}$ & 2005 -El Peligro y El Pato & 25 productores \\
\hline $\begin{array}{l}\text { Cooperativa Agropecuaria } \\
\text { Nueva Esperanza Limitada }\end{array}$ & 2011 - Abasto, Olmos y Etcheverry & $\begin{array}{l}30 \text { familias de productores y } \\
\text { medieros hortícolas, } \\
\text { arrendatarios }\end{array}$ \\
\hline $\begin{array}{l}\text { Cooperativa Agropecuaria } \\
\text { Productores Parque Pereyra }\end{array}$ & 2001 - Parque Pereyra & 60 productores hortícolas \\
\hline $\begin{array}{l}\text { Cooperativa de Trabajo La } \\
\text { Esperanza }\end{array}$ & 2013 - El Pato & $\begin{array}{l}20 \text { pequeños productores } \\
\text { hortícolas }\end{array}$ \\
\hline $\begin{array}{l}\text { Cooperativa Las Acacias } \\
\text { Unidas }\end{array}$ & 2013 - Berazategui y El Peligro & 32 productores \\
\hline $\begin{array}{l}\text { Cooperativa Moto Méndez de } \\
\text { Horticultores Platenses } \\
\text { Limitada }\end{array}$ & $\begin{array}{l}2012 \text { - Lisandro Olmos, Abasto y Ángel } \\
\text { Etcheverry }\end{array}$ & 45 productores \\
\hline Cooperativa Unión Renovada & 2012 - El Pato, El Peligro & 55 productores \\
\hline $\begin{array}{l}\text { CoTrAHyP, Cooperativa de } \\
\text { Trabajo Agropecuario de } \\
\text { Limitada }\end{array}$ & 2005 - Hudson y Pereyra & 30 productores \\
\hline Cooperativa Unión y Fuerza & 2014 - Florencio Varela y Almirante Brown & 30 productores diversificados \\
\hline $\begin{array}{l}\text { Los Amigos Reserva de } \\
\text { Biósfera de Pereyra }\end{array}$ & $\begin{array}{l}2011 \text { - Parque Pereyra, Villa Elisa y } \\
\text { Berazategui }\end{array}$ & $\begin{array}{l}13 \text { productores, en su totalidad, } \\
\text { hortícolas }\end{array}$ \\
\hline
\end{tabular}




\begin{tabular}{|l|l|l|}
\hline $\begin{array}{l}\text { Movimiento de Pequeños } \\
\text { Productores (MPP) }\end{array}$ & $\begin{array}{l}2015 \text { - Abasto, Ángel Etcheverry, Los } \\
\text { Hornos, Olmos, Poblet, Arana }\end{array}$ & 400 familias \\
\hline $\begin{array}{l}\text { Unión de Trabajadores de la } \\
\text { Tierra (UTT) }\end{array}$ & $\begin{array}{l}\text { 2010 - Varela, El Pato, El Peligro, Colonia } \\
\text { Urquiza, Los Porteños, Las Banderitas, } \\
\text { Melchor Romero, Abasto, Etcheverry, } \\
\text { Luján, Cañuelas, San Vicente }\end{array}$ & 500 \\
\hline
\end{tabular}

Para ello, fue fundamental poner en valor el trabajo que venían realizando distintos técnicos en los territorios, los cuales se vincularon con municipios y universidades, entre otras instituciones. Su anclaje y articulación con los niveles locales permitió la generación de programas de territorio en los que los ejes fundamentales son producción y comercialización, soberanía alimentaria y equidad social, contaminación ambiental, y aportes al ordenamiento territorial (Barsky y Aboitiz, 2011).

\section{Gráfico 1: Origen de las organizaciones}

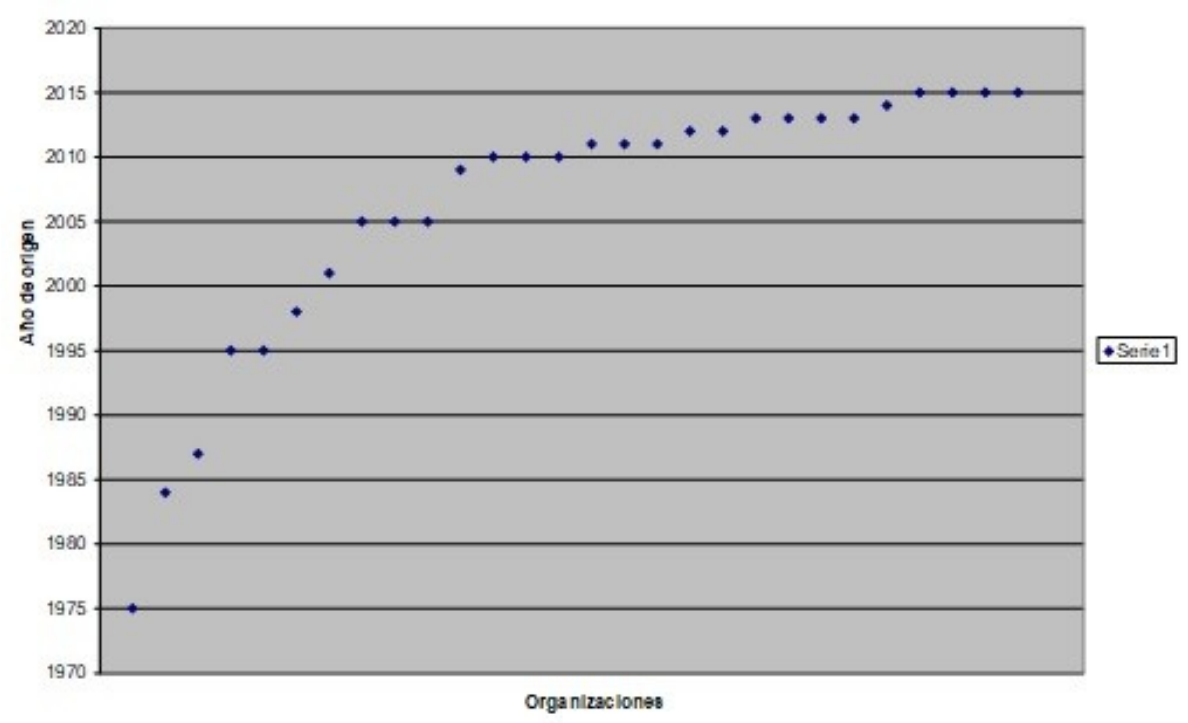

En el gráfico 1 se puede visualizar que entre los años 2010 y 2015 se originaron la gran mayoría de las organizaciones señaladas en la Tabla 2.

A fines del año 2012 se conformó la Mesa Regional de Pequeños y Medianos Productores Agropecuarios, que en un principio reunió a 26 organizaciones. En la actualidad, participan de ella 22 organizaciones.

La Mesa comenzó a partir de una iniciativa de los productores, y avanzó como un espacio en el que se reunieron las organizaciones con el fin de solicitar apoyo económico a las instituciones del Estado en forma de subsidios por problemas climáticos (emergencia climática). En la actualidad es un espacio de referencia para los funcionarios políticos de diferentes estamentos al momento de negociar medidas políticas que incumben al sector.

La conformación de la mesa es parte de un proceso en el que circulan distintas visiones en cuanto a temáticas de interés común, entre ellas el acceso a la tierra y la comercialización.

\subsection{La tierra y comercialización como problemáticas}

El desarrollo de los periurbanos como parte de la expansión de las grandes ciudades ha provocado el despliegue de procesos y disputas por el acceso a la tierra. Esta situación se visualiza en los partidos del AMBA Sur.

Así, tanto los valores de compra como de arrendamiento registran un pico tan elevado que complica la actividad 
productiva. Alrededor del $90 \%$ de los productores de la AF son arrendatarios: “...para nosotros siempre un eje fundamental fue la lucha por la tierra, desde el primer momento, desde el primer plenario en la UTT, siempre se planteó la temática de la tierra, ahí se empezaron a hacer acciones...” (Entrevista a referente de la UTT, diciembre 2015)

La fuerte devaluación registrada en Argentina en diciembre del año 2015, unida al incremento en los valores del arrendamiento, las tarifas de servicios eléctricos y el valor de los insumos en dólares, ha provocado que se llegue a una situación de recurrentes reclamos para visibilizar las problemáticas.

Otro de los reclamos está relacionado con la ley de Agricultura Familiar, que contempla la problemática de la tierra, la cual, si bien fue sancionada en el año 2014, aún no fue reglamentada.

La comercialización de la producción hortícola se realiza por dos circuitos, el directo (productor-consumidor), con varias modalidades como ferias, entregas a domicilio, mercados populares, etc., y el circuito tradicional indirecto en el que interviene una mayor cantidad de eslabones hasta llegar al consumidor.

Más del 95 \% de la producción hortícola de los productores familiares se comercializa por el circuito indirecto, en una modalidad que se denomina vulgarmente "culata de camión" donde el "camionero" (intermediarioconsignatario) encarga la mercadería y la retira en el plazo acordado, dejando previamente los cajones "vacíos” en la quinta en la que realizó el pedido. Por lo general los vacíos se entregan en el momento de retirar otra carga. Los “camioneros”, llevan la mercadería a diferentes mercados concentradores, como los de La Plata, Buenos Aires, Rosario, Córdoba, etc. (García et al, 2011; Waisman, 2012).

La posibilidad de participar en espacios de comercialización directa permite a los productores hortícolas familiares apropiarse de un mayor margen de comercialización a partir de la obtención de precios hasta un $500 \%$ mayores a los obtenidos en culata de camión. Durante la consolidación de estas experiencias las familias siguen dependiendo de las formas tradicionales de comercialización (principalmente a culata de camión), ya que la frecuencia y el volúmen que comercializan en estos canales no son suficientes para el total de lo que producen (Bravo y Ferraris, 2013).

La problemática de la comercialización es sentida por las organizaciones de la agricultura familiar y la enfrentan según sean las particularidades de la misma, siempre en procura de la disminución de la intermediación a través de diferentes estrategias: “...trabajamos en buscar canales de comercialización más cercanos al consumidor, más dinámicos. Lograr ventas al Estado...” (Entrevista a referente de la UTT, diciembre 2015)

En cuanto a la comercialización de flores, también se han buscado caminos alternativos, como ya lo mencionamos anteriormente sobre la instalación de MERCOFLOR.

\section{Consideraciones finales}

El contexto de las políticas implementadas desde el Estado hacia el sector de la Agricultura Familiar, tanto en su órbita nacional como provincial y municipal, hasta 2015, promovieron instancias que facilitaron la organización en procesos que apuntaron a alcanzar una participación genuina, y revirtieron el diagnóstico realizado por el INTA en el año 2005 . En la actualidad se puede constatar la importante presencia de las organizaciones en mesas regionales y provinciales, que generan y fortalecen instancias organizativas territoriales cada vez más articuladas. Las organizaciones articulan entre sí, con funcionarios del Estado y con organismos privados relacionados con la actividad hortiflorícola.

Se han consolidado espacios de discusión en los que las organizaciones demandan e interpelan al poder político, y exigen al Estado que use su poder ordenador en relación con el accionar de los diferentes actores privados concentradores del poder económico, los cuales marcan los lineamientos de las condiciones de producción y comercialización. 
A través del accionar de las organizaciones se visibiliza la importancia social y económica del sector productivo en la región.

El conflicto está presente; el contexto económico vigente desde fines del año 2015 ha propiciado la presencia de los productores organizados en las calles, debido al fuerte retroceso económico general que ha provocado la importante disminución de los ingresos globales de las familias productoras. Coyunturalmente reclaman por el incremento de tarifas, por los servicios y los alquileres. Asimismo, este importante nivel de organización hace posible canalizar los reclamos históricos relacionados con mejores condiciones de vida y producción, y fundamentalmente con el acceso a la tierra.

\section{Notas}

1 CEDEPO : Centro Ecuménico de Educación Popular

\section{Bibliografía}

Barsky, A. y AboitiZ, P. (2011). La agricultura periurbana en la agenda pública. Implementación de políticas municipales, provinciales y nacionales para el Sostenimiento del cinturón verde en los bordes de la Región Metropolitana de Buenos Aires (2000-2010). Jornadas “Memoria y oportunidades en el agro argentino: burocracia, tecnología y medio ambiente (1930-2010)”, Universidad Nacional de General San Martin. Recuperado de http://www.ungs.edu.ar/ms ici/wp-content/uploads/2012/11/Barsky-Aboitiz.pdf (pp.1.-3)

Barsky,A. (2013). Gestionando la diversidad del territorio periurbano desde la complejidad de las instituciones estatales. Implementación de políticas públicas para el sostenimiento de la agricultura en los bordes de la Región Metropolitana de Buenos Aires (2000-2013) (Tesis Doctoral), Universidad Autónoma de Barcelona, España. En línea http://ddd.uab.cat/record/118629

Bertoni, L. y Soverna, S. (noviembre 2014). La transición en las políticas públicas para la agricultura familiar Argentina de los programas a la nueva institucionalidad. XVII Jornadas Nacionales de Extensión Rural y IX del Mercosur El encuentro en la diversidad, Zavalla - Santa Fe. Recuperado de: https://onedrive.live.com/? cid=2d37fcdea19f0f15\&id=2D37FCDEA19F0F15\%21192\&authkey=\%21AAqAUM9 1WMQELw

Boletín Oficial de la República Argentina Decreto 1030. Recuperado de https://ar.vlex.com/vid/decreto$\underline{518081038}$

Cardarelli, G. y Rosenfeld, M. (1998). Las participaciones de la pobreza. Buenos Aires: PAIDOS.

Dumrauf, S., Giordano, G., Marasas, M., Moreyra, A., Prividera, G., Tito, G. (2012). Los procesos participativos para la construcción de líneas de investigación. Ediciones INTA. Recuperado de: http://inta.gob.ar/documentos/los-procesos-participativos-para-la-construccion-de-lineas-de-investigacion

Feito, M. C. (2005). Evaluación de la implementación del programa Cambio rural en el área hortícola bonaerense: operatoria, logros obtenidos y cuestiones pendientes. En R. Benencia, y C. Flood, (coord.), Trayectorias y contextos. Organizaciones rurales en la Argentina de los noventa. Edit. La Colmena. Bs. As.

Ferraris, G. y Bravo, M. (diciembre 2014). Organizaciones de productores hortícolas del Cinturón Verde de La Plata. VIII Jornadas de Sociología de la UNLP. Ensenada, Argentina. Recuperado de: http://www.memoria.fahce.unlp.edu.ar/trab eventos/ev.4751/ev.4751.pdf

Foro Nacional de Agricultura Familiar FONAF (2006). Segundo Plenario. Recuperado de: http://www.fonaf.org.ar/documentos/Documento Mendoza mayo 2006.pdf 
Fournier, M., Hantouch, J., Spampinato, S., Testa, D., y Garcia, O (2012). Diplomatura en Fortalecimiento de las Capacidades de Gestión de Organizaciones Sociales Territoriales. Módulo 2 Gestión de Organizaciones Sociales. Recuperado de: http://www.cenoc.gob.ar/documentos/diplo2.pdf Consultado 3 de septiembre de 2013

García, M. y Kebat, C. (2008). Transformaciones en la horticultura platense. Una mirada a través de los censos. Realidad económica, 237, 110-134.

García, M., Le Gall, J., y Mierez, L. (2011). Comercialización tradicional de hortalizas de la Región Metropolitana Bonaerense. Herencias, dinámicas e innovaciones de un sistema complejo. Boletín Hortícola, 47.

García, M. (2008). Uso y acceso a la tierra en el marco del nuevo modelo productivo de la horticultura platense. Revista Interdisciplinaria de Estudios Agrarios (RIEA), 29. $2^{\circ}$ semestre.

Hindi, G. (2015). Resignificaciones del cooperativismo en el marco de la expansión de políticas de Economía Social. Revista Idelcoop, 216, $11-\quad 25 . \quad$ Recuperado de http://www.idelcoop.org.ar/sites/default/files/revista/articulos/pdf/revista-216-ref-y-deb-01.pdf

INTA (2005). Plan tecnológico regional 2006-2008 informe diagnóstico de situación. Cadena Hortícola. Recuperado de: www.funcex.org.br/material/REDEMERCOSUL.../ARG 170.pdf

INTA (2008). PTR 2009 - 2011 CRBAN. Informe etapa de diagnóstico en profundidad de Territorios. Agencia Extensión Rural de La Plata.

IPAF Región Pampeana INTA (2012). Foro de Universidades para la Agricultura familiar. Documento 1. Recuperado de: file:///E:/biblio/FORO-UNIVERSIDADES FINAL-MIERCOLES-4-1.pdf

Melucci, A. (1999). Teoría de la acción colectiva. En Acción Colectiva, Vida Cotidiana y Democracia. El Colegio de México (pp. 25-54 Recuperado de: http://www.ses.unam.mx/docencia/2016II/Melucci1999 AccionColectivaVidaCotidianaYDemocracia.pdf

Merchán, A. (2016). Valorización de la Tierra en el Cinturón Hortícola Platense. Disparidad en el Valor de los Arrendamientos. Maestría PLIDER (FCAyF-UNLP).

Programa Cambio Rural- Manual de Procedimientos. Ciudad Autónoma de Buenos Aires. Octubre de 2010. pp. 4:6. Recuperado de: http://procadisaplicativos.inta.gob.ar/cursosautoaprendizaje/espacio/profeder/docs/manual procedimientos cambi o rural.pdf

Robirosa, V., Cardarelli, G., y Lapalma, A. (1995). Planificación y Turbulencia. UNICEF SIGLO XXI, EspañaBuenos Aires, Argentina.

RInguelet, R. (2008). La complejidad de un campo social periurbano centrado en la zonas rurales de La Plata. Revista Mundo Agrario, 17. Segundo semestre.

Ringuelet, R. y Garat, J. J. (2000). Los cambios de los sectores productivos tradicionales en la horticultura platense y sus formas asociativas. En Roberto Ringuelet, R. (2000). Espacio tecnológico, población y reproducción social en el sector hortícola de La Plata En línea. La Plata: UNLP.FaHCE (EstudiosInvestigaciones, 39). Recuperado de: http://www.memoria.fahce.unlp.edu.ar/libros/pm.182/pm.182.pdf

Seibane, C., Larrañaga, G., Kebat, C., Hang, G., Ferraris, G., Bravo, M. L. (2014). Redes para la promoción del desarrollo territorial en el cinturón hortícola platense. Reflexiones y aportes. Mundo Agrario, 15(29) ,1-19. Recuperado de: http://www.mundoagrario.unlp.edu.ar/article/view/4239

SENASA (2016). Comisión de Agricultura Familiar de Senasa SENAF. Recuperado de http://www.senasa.gov.ar/informacion/agricultura-familiar/senaf 
Valtriani, A. y Velarde, I. (2001). La Asociación de Medieros Hortícolas de La Plata: de la reivindicación a la organización de tipo económico. II Jornadas de Estudios agrarios y agroindustriales. Facultad de Ciencias Económicas. UBA. Bs As.

Vega, M. (1999). Integración vertical y productos diferenciados. Boletín Hortícola, Año 7, 23.

Waisman, M. A. (2012). Dime a quién le vendes y te diré quién eres...Relaciones entre actores relevantes y dinámica histórica en la comercialización de hortalizas en el periurbano de la ciudad de La Plata. En: Actas de las Jornadas Académicas Tierra y Movimientos Sociales en la Argentina. "A cien años del Grito de Alcorta”, Universidad Nacional de Rosario, Facultad de Humanidades y Artes, Rosario, 29, 30 y 31 de Agosto, ISBN: 978987-677-049-1. 